\title{
Cryptography for Unconditionally Secure Message Transmission in Networks (Invited Talk)
}

\author{
Kaoru Kurosawa \\ Ibaraki University, Japan \\ kurosawa@mx.ibaraki.ac.jp
}

We consider the model of unconditionally secure ( $r$-round, $n$-channel) message transmission schemes which was introduced by Dolev et al. [1]. In this model, there are $n$ channels between a sender and a receiver, and an infinitely powerful adversary $\mathbf{A}$ may corrupt (observe and forge) the messages sent through $t$ out of $n$ channels. The sender wishes to send a secret $s$ to the receiver in $r$-round without sharing any key with the receiver.

We say that a message transmission scheme is perfectly secure if it satisfies perfect privacy and perfect reliability. The perfect privacy means that the adversary A learns no information on $s$, and the perfect reliability means that the receiver can output $s$ correctly. We say that it is almost secure if it satisfies perfect privacy and almost reliability.

In this talk, we survey some protocols and bounds for the above problem. We also describe some new results for general adversary structures 4.5. In particular, we introduce the general error decodable secret sharing scheme and show its application to 1-round perfectly secure message transmission schemes [4].

\section{References}

1. Dolev, D., Dwork, C., Waarts, O., Yung, M.: Perfectly Secure Message Transmission. J. ACM 40(1), 17-47 (1993)

2. Kurosawa, K., Suzuki, K.: Almost Secure (1-round, n-channel) Message Transmission Scheme. In: Desmedt, Y. (ed.) ICITS 2007. LNCS, vol. 4883, pp. 99-112. Springer, Heidelberg (2009); Also appeared in Cryptology ePrint Archive: Report 2007/076 (2007)

3. Kurosawa, K., Suzuki, K.: Truly Efficient 2-Round Perfectly Secure Message Transmission Scheme. IEEE Transactions on Information Theory 55(11), 5223-5232 (2009)

4. Kurosawa, K.: General Error Decodable Secret Sharing Scheme and Its Application. Cryptology ePrint Archive: Report 2009/263 (2009)

5. Kurosawa, K.: Round-Efficient Perfectly Secure Message Transmission Scheme against General Adversary. Cryptology ePrint Archive: Report 2010/450 (2010) 